\title{
Les Mollusques du lac de Tignes (Savoie) : intérêt de ce groupe faunistique pour caractériser la qualité biologique des sédiments des lacs d'altitude
}

\section{J. Mouthon 1}

Mots clés : Mollusques, eaux stagnantes, qualité biologique, pollution.

Un inventaire qualitatif et quantitatif des mollusques des zones littorales et profondes du lac de Tignes, a permis de dresser une cartographie détaillée des secteurs pollués de ce plan d'eau.

The Mollusca of Tignes lake (Savoie) : their usefulness in characterising water quality in high altitude lakes.

Keywords: Mollusca, standing waters, water quality, pollution.

A quantitative and qualitative inventory of the littoral and profundal Mollusca of Tignes lake allows polluted areas to be readily delimited.

\section{I. - Introduction}

Généralement peu exploités jusqu'à ces dernières années, les plans d'eau d'altitude suscitent à l'heure actuelle un intérêt accru de la part des municipalités, qui voient dans leurs attraits touristiques et halieutiques l'espoir d'un nouveau développement.

Soucieux d'apporter aux organismes gestionnaires les éléments nécessaires à leur politique, nous voulons présenter, dans le cas du lac de Tignes, l'intérêt et la contribution que peut apporter l'étude des malacocénoses à la connaissance de la qualité biologique des sédiments de ce type d'écosystème.

\section{2. - Le milieu}

Le lac de Tignes est situé à une altitude de $\mathbf{2 0 8 6}$ $\mathrm{m}$. dans le département de la Savoie, au sud-ouest du village du même nom, et de la retenue hydroélectrique du Chevril.

Estimée à 32 hectares, au début du siècle (Delbecque 1898 ), sa superficie a été notablement réduite

1. CEMAGREF 3, Quai Chatveau 69009 LYON, France. par les travaux d'aménagements du site (creusement de l'exutoire, comblement des zones marécageuses) et n'est plus actuellement que de 24 ha.

Mal connue, sa bathymétrie se caractérise par la présence de 3 fosses (fig. 1) dues, d'après Delbecque (op. cit.) « à des effondrements du fond originel dans des cavités creusées par les eaux souterraines ".

Gelé de novembre à mai, ce plan d'eau classé dans la catégorie des lacs dimictiques duler ordre, reçoit les eaux de deux afférences, l'une longeant le terrain de golf dans son cour inférieur, l'autre, appelé "ruisseau du Val Claret " drainant les eaux pluviales de la station de Tignes (Super-Tignes).

Les eaux alcalines et fortement minéralisées du lac et de ses affluents présentent des concentrations en sulfates de calcium et de magnésium particulièrement élevées, liées essentiellement à la nature géologique du bassin versant (Tableau I).

Cette particularité confère à ce plan d'eau une évidente fragilité puisqu'un déficit en oxygène dissous au voisinage des fonds, entraînerait rapidement une réduction des sulfates en hydrogène sulfuré très toxique. Toutefois ce ne semble pas être encore le cas, en effet les mesures effectuées au cours du mois d'aoùt 1979, montrent que le taux de saturation demeure relativement élevé jusqu'au fond du lac (Tableau I). 


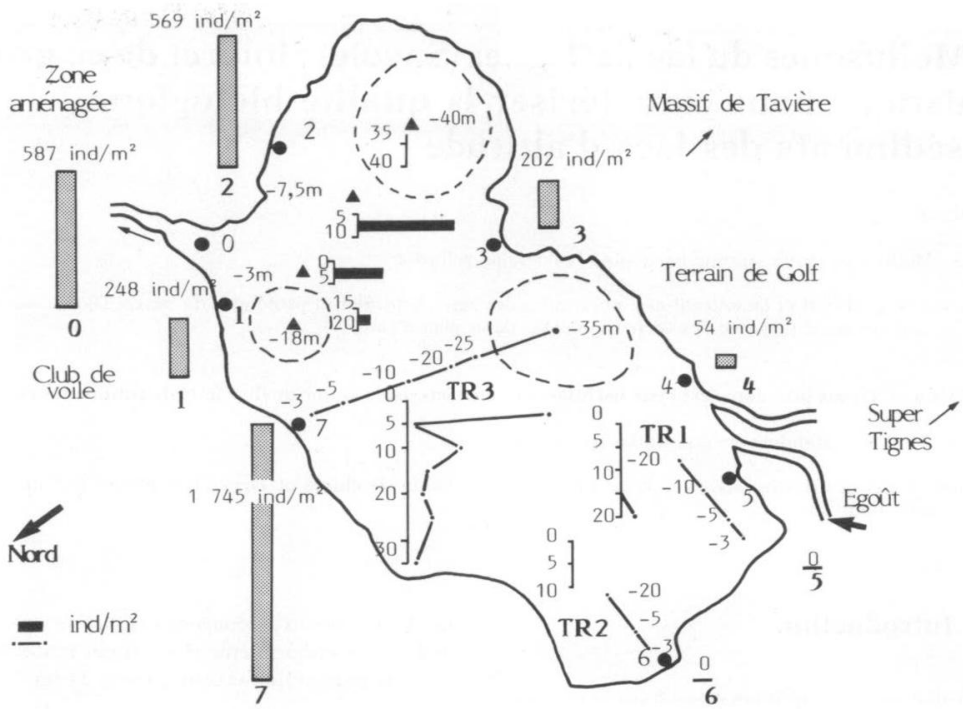

Fig. 1: Distribution bathymétrique et répartition de l'abondance des especes de mollusques dans les zones littorale et profonde du lac de Tignes.

La végétation est essentiellement composée de bryophyte: Brachytecium rivulare, et d'algues: Chara fragilis, Hydrurus faetidus, Vaucheria sp., Zygogonium sp.

\section{3. - Techniques d'échantillonnage}

Les mollusques de la zone littorale et de l'affé. rence ont été récoltés à l'aide d'un troubleau à base rectangulaire (Long. $=25 \mathrm{~cm}$, Haut. $=18 \mathrm{~cm}$ ) L'échantillonnage réalisé sur toute la largeur de la zone lit torale représente une surface totale de 1,2 . parfois $3 \mathrm{~m}^{2}$ par station. Les zones profondes ont été prospectées à l'aide d'une Benne Petersen (surface $=350 \mathrm{~cm}^{2}$ ), trois à six prélèvements étant effectués à chaque point d'échantillonnage.

L'abondance des espèces prélevées est ensuite rapportée au $\mathrm{m}^{2}$.
Les échantillons furent tamisés à $630 \mu$, puis triés et déterminés sous la loupe binoculaire.

Les prélèvements réalisés au cours du mois de juillet 1984 concernent 24 points répartis dans les zones littorales et profondes de ce lac.

\section{4. - Les Malacocénoses}

\section{1. - Remarques sur les peuplements}

Grâce aux recherches de Kuiper (1974), les malacocénoses littorales des lacs d'altitude de la région sont relativement bien connues. En revanche, les données concernant les peuplements profonds de ces milieux sont plutôt rares (Mouthon 1981).

Parmi les 4 espèces de mollusques recensées dans le lac de Tignes, les deux gastéropodes Lymnaea peregra, $L$. truncatula, et le bivalve Pisidium 
Tableau I. Résultats des analyses physico-chimiques des eaux du lac de Tignes et de ses afférences. Prélèvements du 14/08/1979.

\begin{tabular}{|c|c|c|c|c|c|c|c|c|c|c|c|}
\hline \multirow[b]{2}{*}{ Eléments } & \multicolumn{8}{|c|}{ Lac } & \multirow{3}{*}{$\frac{\begin{array}{c}\text { Ruisseau } \\
\text { du } \\
\text { Golf }\end{array}}{7}$} & \multirow{2}{*}{$\begin{array}{c}\text { Ruisseau } \\
\text { de } \\
\text { * Valclaret }\end{array}$} & \multirow{2}{*}{$\begin{array}{l}\text { Egout } \\
\text { afférent du } \\
\text { "Valclaret }\end{array}$} \\
\hline & Surface & $5 \mathrm{~m}$ & $10 \mathrm{~m}$ & $15 \mathrm{~m}$ & $25 \mathrm{~m}$ & $30 \mathrm{~m}$ & $35 \mathrm{~m}$ & $37,5 \mathrm{~m}$ & & & \\
\hline Température ${ }^{\circ} \mathrm{C}$ & 9,9 & 8,6 & 7,5 & 4,8 & 4,4 & 4,4 & 4,4 & 4,3 & & 3 & 10 \\
\hline Ph & 7,7 & 7,9 & 7,7 & 7,7 & 7,7 & 7,7 & 7,7 & 7,7 & 7,9 & 7,8 & 7,5 \\
\hline Conductivité $\mu \mathrm{s}$ & 840 & 890 & 1050 & 1090 & 1100 & 1100 & 1100 & 1100 & 680 & 1050 & 540 \\
\hline $\mathrm{O}_{2}$ dissous $\mathrm{mg} / \mathrm{l}$ & & & & & & & & & & & \\
\hline (saturation) & 8,7 & 8,9 & 8,9 & 9,8 & 9,9 & 9,9 & 9,9 & 10 & & & \\
\hline $\begin{array}{l}\mathrm{O}_{2} \text { dissous } \mathrm{mg} / \mathrm{l} \\
\text { (terrain) }\end{array}$ & 9,9 & 11,2 & 9,7 & 8,1 & 7,2 & 7,4 & 7,2 & 7,5 & & & \\
\hline Taux de saturation \% & 114 & 126 & 109 & 83 & 73 & 75 & 73 & 75 & & & \\
\hline Oxydabilité mg/l & 0,00 & 0,03 & 0,11 & 0,21 & 0,29 & 0,19 & 0,29 & 0,36 & 0,31 & 0,09 & 7,93 \\
\hline $\begin{array}{l}\text { Azote ammoniacale } \\
\mathrm{mg} / \mathrm{N}\end{array}$ & 0,01 & 0,01 & 0,03 & 0,03 & 0,02 & 0,01 & 0,03 & 0,03 & 0,01 & 0,01 & 4,88 \\
\hline Azote nitreux mg/l N & 0.00 & 0.00 & 0,00 & 0,00 & 0,00 & 0,00 & 0,00 & 0,00 & 0,00 & 0,00 & 0,00 \\
\hline Azote nitrique mg/l N & 0,15 & 0,14 & 0.13 & 0,15 & 0,15 & 0,15 & 0,16 & 0,18 & 0,15 & 0,18 & 0.01 \\
\hline Orthophosphates mg/l P & 0,00 & 0,00 & 0,00 & 0,00 & 0,00 & 0,00 & 0,00 & 0,00 & 0,00 & 0.00 & 1,5 \\
\hline Hydrophosphates mg/l P & 0,01 & 0,01 & 0,01 & 0,01 & 0,01 & 0,00 & 0,02 & 0,01 & 0,01 & 0,00 & 0,00 \\
\hline Silice soluble $\mathrm{SiO}_{2} \mathrm{mg} / \mathrm{l}$ & 4,40 & 4,60 & 4.90 & 6,00 & 6,30 & 6.40 & 6.40 & 6,40 & 4,80 & 5,20 & 7.60 \\
\hline $\mathrm{HCO}_{3} \cdot \mathrm{mg} / \mathrm{l}$ & 98 & 98 & 98 & 98 & 98 & 98 & 104 & 98 & 98 & 92 & 177 \\
\hline $\mathrm{Cl}-\mathrm{mg} / \mathrm{l}$ & 1,50 & 1,00 & 1,50 & 1,20 & 1,50 & 1,30 & 1,30 & 1,50 & 1,00 & 1.10 & 10,5 \\
\hline $\mathrm{SO}_{4} \mathrm{mg} / \mathrm{l}$ & 450 & 500 & 600 & 650 & 600 & 650 & 650 & 650 & 350 & 600 & 150 \\
\hline $\mathrm{Ca}^{++} \mathrm{mg} / \mathrm{l}$ & 176 & 192 & 232 & 244 & 244 & 248 & 248 & 248 & 136 & 232 & 72 \\
\hline $\mathrm{Mg}^{++} \mathrm{mg} / \mathrm{l}$ & 29,7 & 28,0 & 35,9 & 35,9 & 37.6 & 26,7 & 26.5 & 26.5 & 15,8 & 24.9 & 8.1 \\
\hline $\mathrm{Na}^{+}+\mathrm{mg} / \mathrm{l}$ & 1,40 & 1,90 & 2,40 & 2,5 & 2,6 & 2,4 & 2,4 & 2,5 & 1,7 & 2,4 & 12,8 \\
\hline $\mathrm{K}^{+} \mathrm{mg} / \mathrm{l}$ & 0,40 & 0,40 & 0.40 & 0,4 & 0,5 & 0.4 & 0,4 & 0,5 & 0,3 & 0,4 & 5,2 \\
\hline
\end{tabular}

casertanum sont inféodés à la zone lit torale ; tandis que Pisidium personatum que l'on retrouve également dans les vases de bordure au niveau de la con. fluence de ruisseaux afférents (Point 4, fig. 1), et de l'exutoire (Point 0 ), est la seule espèce qui colonise les zones sublittorales et profondes (Tableau II).

Absente semble-t-il des plans d'eau d'altitude pyrénéens, cette espèce est peu répandue dans les lacs haut-alpins (Kuiper, op. cit.).

\section{2. - Distribution des especes dans la zone littorale.}

Particulièrement sensibles aux modifications du milieu (Mouthon, op. cit.) notamment dans les écosystèmes d'altitude où règnent des conditions extrèmes, les malacocénoses du lac de Tignes présentent des variations de densité remarquables d'un point d'échantillonnage à l'autre (fig. 1).

C'est au niveau des bordures septentrionales et orientales que ces abondances sont les plus élevées
(Points 0,1, 2 et 7). Cependant la prolifération de Pisidium casertanum au point 7 semble être due à l'arrivée d'apports organiques diffus, pour l'heure plus eutrophisants qu'inhibiteurs. En revanche la diminution de la densité observée au point 1, est probablement liée aux activités du Club de Voile installé à proximité.

Affectés par un déversement accidentel d'hydrocarbures, et par l'arrivée des égouts du « Val Claret " drainant les eaux pluviales de la station de Super-Tignes, les mollusques sont absents de toute la bordure occidentale du lac (points 5 et 6 ).

A l'aval du ruisseau du "Golf " (point 4) les mollusques réapparaissent, mais la densité des peuplements, à nouveau perturbée par les traitements nécessaires à l'entretien du terrain de golf, demeure toutefois remarquablement faible. Ce n'est qu'à l'extrémité de la bordure orientale du lac (point 3) que les malacocénoses atteignent des abondances plus conséquentes, témoignant d'une amélioration notable de la qualité des sédiments. 
Tableau II : Abondance des espèces aux différents points d'échantillonnage.

\begin{tabular}{|c|c|c|c|c|c|c|c|c|c|c|c|c|c|c|c|c|c|c|c|c|}
\hline \multirow[b]{3}{*}{ Points } & \multicolumn{8}{|c|}{ Zone littorale } & \multicolumn{12}{|c|}{ Fonds } \\
\hline & \multirow[b]{2}{*}{0} & \multirow[b]{2}{*}{1} & \multirow[b]{2}{*}{2} & \multirow[b]{2}{*}{3} & \multirow[b]{2}{*}{4} & \multirow[b]{2}{*}{5} & \multirow[b]{2}{*}{6} & \multirow[b]{2}{*}{7} & \multicolumn{2}{|l|}{$\mathrm{TR}_{\mathrm{I}}$} & \multirow[b]{2}{*}{-5} & \multirow[b]{2}{*}{-10} & \multicolumn{2}{|c|}{$\mathrm{TR}_{3}$} & \multirow[b]{2}{*}{-25} & \multirow[b]{2}{*}{-35} & \multirow[b]{2}{*}{$\begin{array}{l}-18 \\
m\end{array}$} & \multicolumn{3}{|c|}{ Partie orien } \\
\hline & & & & & & & & & $\begin{array}{l}20 \\
m\end{array}$ & $\begin{array}{l}-3 \\
m\end{array}$ & & & -15 & -20 & & & & -3 & -7.5 & .40 \\
\hline Lymnaea peregra (Müller) & 2 & & 8 & & & & & 1 & & & & & & & & & & & & \\
\hline Lymnaea truncatula Müller & & & 4 & 1 & & & & & & & & & & & & & & & & \\
\hline Pisidium casertanum (Poli) & 405 & 243 & 553 & 201 & 5 & & & 1744 & & & & & & & & & 10 & & & \\
\hline Pisidium personatum Malm & 180 & 5 & 4 & & 49 & & & & 50 & 605 & & 225 & 80 & 60 & 100 & 25 & 35 & 200 & 405 & \\
\hline Nombre sp. & 3 & 2 & 4 & 2 & 2 & - & - & 2 & 1 & 1 & - & 1 & 1 & 1 & 1 & 1 & 2 & 1 & 1 & . \\
\hline Nombre ind $/ \mathrm{m}^{2}$ & 587 & 248 & 569 & 202 & 54 & - & - & 1745 & 50 & 605 & - & 225 & 80 & 60 & 200 & 25 & 45 & 200 & 405 & \\
\hline
\end{tabular}

\section{3. - Distribution bathymétrique}

Généralement inféodée à la zone profonde des écosystèmes lactustres (Favre 1927, 1940, 1941 ; Kuiper 1966...) Pisidium personatum colonise ici tous les compartiments du lac, des bordures jusqu'au niveau des fonds (Tableau II et fig. 1).

En revanche, Pisidium casertanum dont l'amplitude bathymétrique est d'ordinaire plus étendue, demeure cantonnee dans la zone littorale où, à l'exception de quelques rares spécimens récoltés au fond $(-18 \mathrm{~m})$ du fossé d'effondrement appelé "la marmite ", elle ne dépasse pas la profondeur de $3 \mathrm{~m}$.

La prolifération de Pisidium personatum qui " est souvent le seul bivalve présent dans les sources froides et les eaux souterraines surtout dans les régions calcaires " (Kuiper 1966), révèle l'importance de la nappe phréatique dans l'alimentation des eaux du lac.

L'évolution de la distribution bathymétrique de ce Pisidium en différents points du lac montre que :

- les effets inhibiteurs des rejets affectant toute la zone littorale occidentale (points 5 et 6) se font encore sentir au niveau des fonds, totalement dépourvus de mollusques jusqu'à la profondeur de $10 \mathrm{~m}$ (Transects 1 et 2 de la figure 1).

- la présence de population encore abondante de Pisidium personatum jusqu'à la cote de $-35 \mathrm{~m}$ (Transect 3) est révélatrice de la présence de conditions d'oxygenations encore satisfaisantes au niveau des fonds au cours de l'année 1984 (cf. Tableau I).

- malgré l'absence de mollusque dans le fond estimé à environ $40 \mathrm{~m}$, du fossé d'effondrement de la bordure méridionale, la présence de population encore abondante sur tout le plateau précédant l'embouchure de l'exutoire témoigne de la bonne qua. lité des vases dans ce secteur.

\section{5. - Conclusions}

Les variations essentiellement quantitatives des malacocénoses, révélatrices de la qualité des habitats lit toraux et profonds, ont permis de dresser une cartographie des secteurs pertubés du lac de Tignes, comprenant toute la partie occidentale, incluant les fonds jusqu'à $10 \mathrm{~m}$, et près des $2 / 3$ de la bordure méridionale.

Grâce au caractère intégrateur de certains groupes d'invertébrés comme les mollusques, il devient donc possible d'effectuer un diagnostic suffisamment précis et rapide de l'état biologique des sédiments d'un lac, fournissant aux organismes gestionnaires les moyens d'intervenir efficacement sur le milieu.

\section{Travaux cités}

Delebecque (A.). 1868. - Les lacs français. Chamerod et Renouard ed. Paris: $436 \mathrm{p}$.

Favre (J.), 1927. - les Mollusques post-glaciaires du Bassin de Genève. Mèm. Soc. Phys. et Hist. Nat. Genève, 40 (3): 171.434.

Favre (J.h, 1940. - La faune malacologique post-glaciaire et actuelle du lac du Bourget. Annls Ec. Nat. Eaux Forêts, 7 (2): 295-444.

Favre (J.), 1941. - Les Pisidiums du Canton de Neuchatel. Bull. Soc. Neuch. Sci. Nat., $66: 57-112$.

Kuiper (J.G.J.). 1966. - La distribution des espèces vivantes du genre Pisidium C. Pf. en France. J. Conch. $150(4): 181.215$.

Kuiper (J.G.J.). 1974. - Die Pisidien der Hochalpengewässer. Arch. Moll, 104, (1/3) : 1-27.

Mouthon (J.). 1983. - Les Malacocenoses de quatre lacs hautalpins. Annis Jimnol. 19 (2) : 101.106.

Rapport C.T.G.R.E.F., 1979. Lac de Tignes : suivi phvsico-chimique 1978-1979, Ronco, 16 p. 